\title{
The First Manifestation of a Left Atrial Myxoma as a Consequence of Multiple Left Coronary Artery Embolisms
}

\author{
Martin Novak ${ }^{1,2}$, Petr Fila 3 , Ota Hlinomaz ${ }^{1,2}$, Vita Zampachova 4 \\ 1 Department of Cardiology, St. Anne's University Hospital Brno, Pekarska 53, 65691 Brno, Czech Republic \\ 2 International Clinical Research Center, Pekarska 53, 65691 Brno, Czech Republic \\ ${ }^{3}$ Centre of Cardiovascular and Transplantation Surgery, Pekarska 53, 65691 Brno, Czech Republic \\ ${ }^{4}$ Department of Pathology, St. Anne's University Hospital Brno, Pekarska 53, 65691 Brno, Czech Republic
}

\begin{abstract}
A case of multiple embolisms in the left coronary artery as a rare first manifestation of left atrial myxoma is reported. A patient with embolic myocardial infarction and congestive heart failure was treated by percutaneous aspirations and balloon dilatations. Transesophageal echocardiography disclosed a villous myxoma with high embolic potential. Surgical resection of the tumour, suturing of a patent foramen ovale suture and an annuloplasty of the dilated tricuspid annulus was performed the third day after the admission.
\end{abstract}

Recovery of the documented left ventricular systolic function can be explained by resorption of myxomatous material. The patient was discharged ten days after the surgery.

Keywords: heart tumour, myxoma, embolic myocardial infarction, surgical extirpation

Received: 12 May 2017 / Accepted: 4 July 2017

\section{INTRODUCTION}

Myxomas are the most common primary heart tumours most of which occur sporadically. In $90 \%$ of cases it is a solitary tumour, and in $75-90 \%$ of cases, it is localised in the left atrium [1]. The tumour is usually benign and malignant forms of myxomas are very rarely reported [2].

The clinical manifestation of a myxoma depends on its localisation, size and fragility. In the majority of cases, nonspecific systemic symptoms precede from several months up to years before a diagnosis is made. These symptoms are probably caused by the production of interleukin- 6 by tumour cells [3]. Additional possible clinical manifestations such as fatigue, exertional dyspnoea up to pulmonary oedema, vertigo, atrial fibrillation and syncope, following from the obstruction of the mitral orifice and systemic embolisms. Embolisms are described in about one-third of the patients [4]. Right atrial myxomas can cause right-sided heart failure and pulmonary embolism or a paradoxical systemic embolism in case of patent foramen ovale (PFO).

Coronary artery embolism is one of the less frequent clinical manifestations of the tumour.

\section{CASE STUDY:}

A 67 years old man with no significant medical history called an ambulance following a sudden onset of chest pain and shortness of breath which lasted for $20 \mathrm{~min}$ utes.

An ECG showed myocardial infarction with ST elevations in the anterolateral leads. The patient was treated with acetylsalicylic acid $250 \mathrm{mg}$ and heparin 5.000 IU intravenously and transferred for an urgent coronary angiogram. These procedures were started fifty minutes after the onset of chest pain. Embolic occlusions of several branches of the left coronary artery were demonstrated in the periphery of the left an- 
terior descending artery (LAD), the second diagonal artery (D2) and the proximal part of the first obtuse marginal artery (OM1). (Figure 1) There was no pathology relating to the right coronary artery. No signs of pathological vascularization were present. Percutaneous coronary intervention on OM1 was attempted. The occlusion was easily crossed with Runthrough ${ }^{\circledR}$ NS Floppy PTCA guidewire (Terumo, Japan). Repetitive usage of ProntoV4 and Pronto LP extraction catheters (Vascular Solutions, Inc., USA) was unsuccessful, and also dilatations in the site of the occlusion and intracoronary administration of eptifibatide did not lead to the artery recanalization. The only result was a slight movement of embolic mass distally to OM1. (Figure 2) Because there was a clear embolic possibility and minimal effect resulting from aspirations and balloon dilatations in OM1, other occluded coronary artery branches were not percutaneously treated. The patient continued to experience mild chest pain and shortness of breath following the procedure, and he was admitted to the Coronary Care Unit for further therapy.

Transthoracic echocardiography showed a non-dilated left ventricle with akinesia of the apex, lateral wall and part of the inferior wall. Systolic function of the the left ventricle (LV) was moderately impaired with a left ventricle ejection fraction (LVEF) of $40 \%$. The second degree of diastolic LV dysfunction, pseudonormal filling pattern, mild mitral and tricuspid regurgitation,

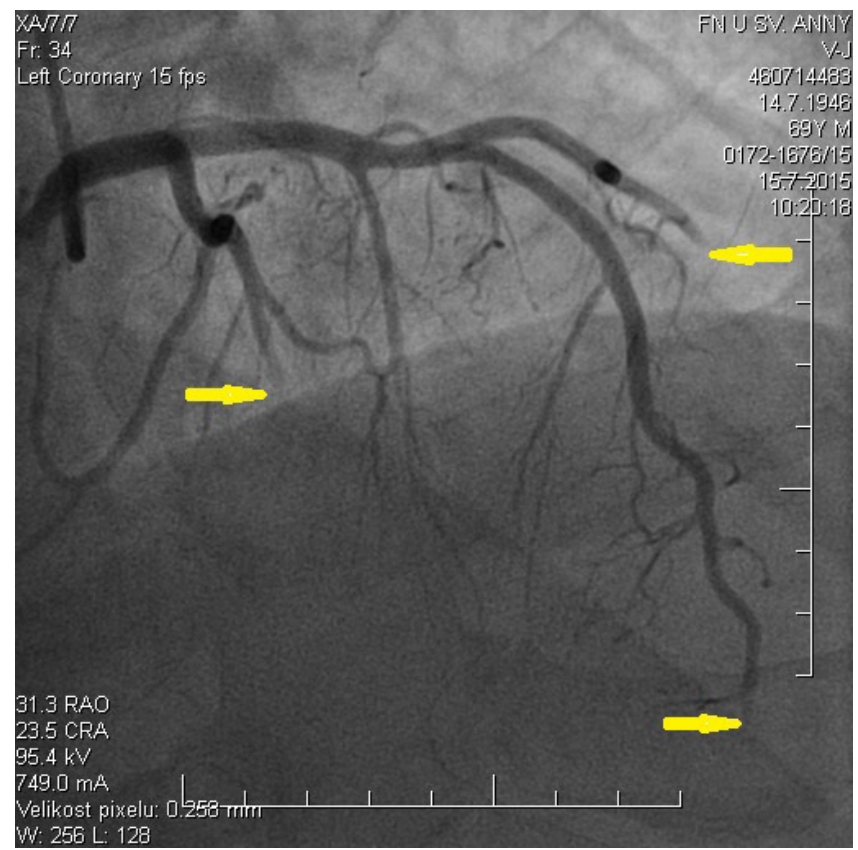

Fig. 1. Embolic occlusion of periphery of LAD, D2, proximal OM1 (arrow) was observed. Other than a mildly enlarged left atrium, no other pathology was found. The right ventricle was not dilated, which showed a well preserved systolic function. Transoesophageal echocardiography was performed subsequently to find the source of the coronary embolism. A $25 \times 20 \mathrm{~mm}$ villous tumour in the left atrium, arising from the interatrial septum, presenting with the echocardiographic features of a cardiac myxoma, was detected. (Figures 3-4) The tumour did not cause an obstruction of the mitral orifice, and the mitral annulus was not dilated. Furthermore, the PFO was recorded as having an insignificant left-to-right shunt. Dilatation of the tricuspid annulus to $47 \mathrm{~mm}$ and moderate tricuspid regurgitation were the other findings. Blood tests showed significant leukocytosis $\left(20 \times 10^{9}\right.$ /1) with mild neutrophilia, C-reactive protein was increased $(113 \mathrm{mg} / \mathrm{l})$. However, procalcitonin levels were within the normal range $(0.12 \mu \mathrm{g} / \mathrm{l})$. The maximum value of creatine kinase $\mathrm{CK}$ was $68 \mu \mathrm{kat} / \mathrm{l}$ and troponin T $5451 \mathrm{ng} / \mathrm{l}$.

At a multidisciplinary team meeting, it was agreed to proceed to with surgery.

A standard pre-assessment was completed and all no contradictory results reported. Neurological examination, including computed tomography (CT) of the brain, excluded embolisms in the intracranial arteries. The patient was treated with intravenous furosemide $120 \mathrm{mg}$ i.v. daily before surgery and also with levosimendan $0,1 \mu \mathrm{g} / \mathrm{kg} / \mathrm{min}$ infusion for 30 hours immedi-

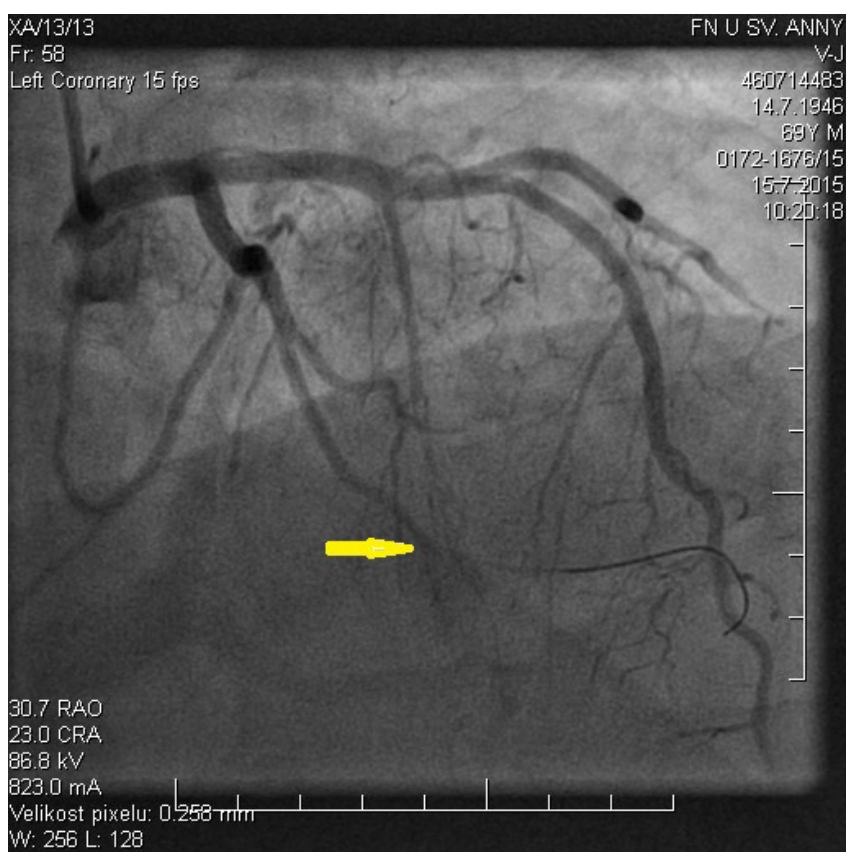

Fig. 2. Result in OM after multiple aspirations and balloon dilatations (arrow) 


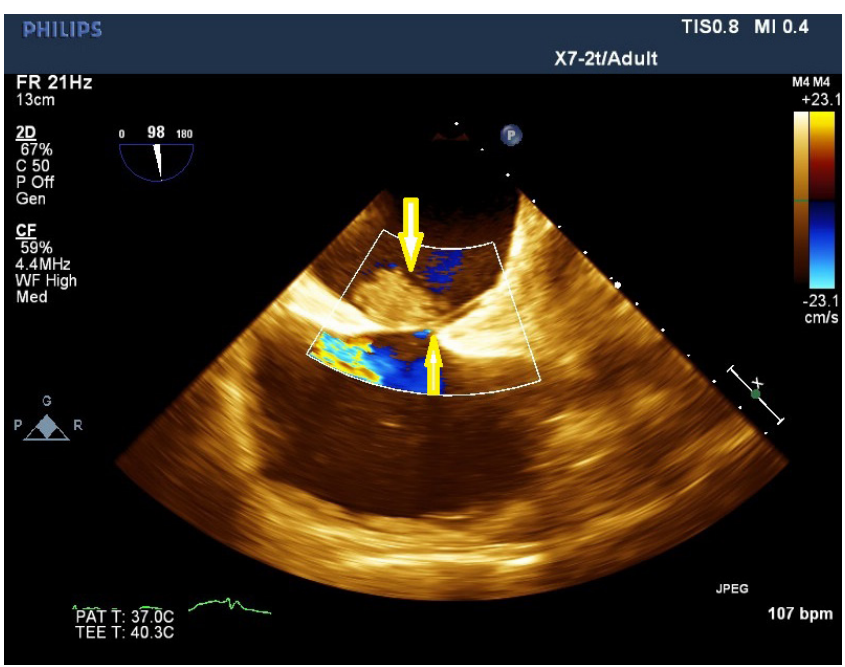

Fig. 3. Left atrial myxoma arising from fossa ovalis area, permanent foramen ovale. 2D TOE view. (arrow)

ately postoperatively due to the development of acute heart failure.

Surgical resection of the tumour was performed the third day after the admission. After the opening of the left atrium, a villous myxoma was removed including the adjacent part of the interatrial septum. (Figures 5-6) The resultant septal defect was closed by direct suturing. Suturing of the PFO and an annuloplasty of the dilated tricuspid annulus was carried out using an Edwards MC3 Tricuspid Annuloplasty Ring (Edwards Lifesciences, USA).

After the surgery, the patient required increased ventilation support and extubation was postponed. There were no other postoperative issues.

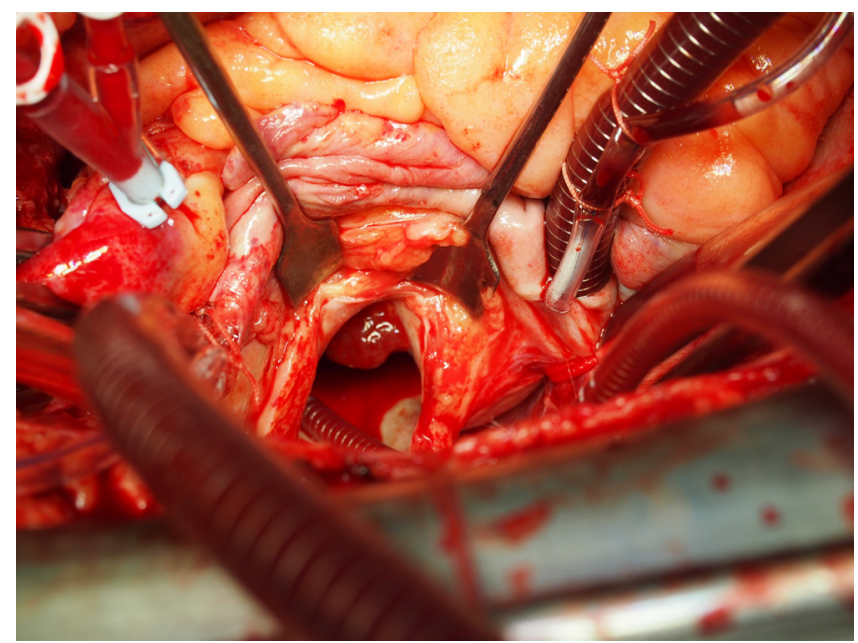

Fig. 5. Surgical view into the left atrium with myxoma arising from interatrial septum

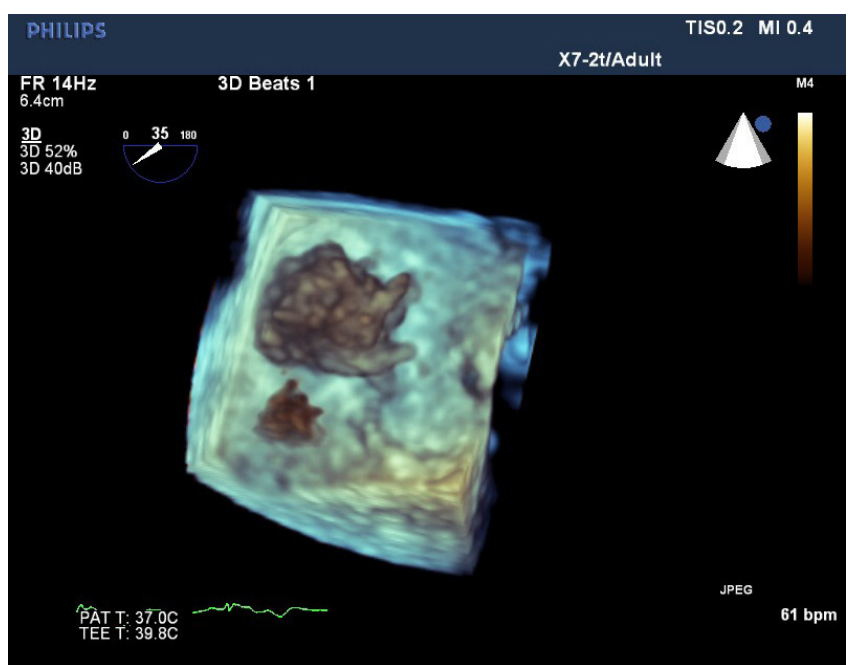

Fig. 4. Villous left atrial myxoma. 3D TOE view

Histology confirmed the diagnosis of a left atrial myxoma (Figures 7-8) with typical findings of stellate cells with eosinophilic cytoplasm in a myxoid stroma, extravasated erythrocytes and hemosiderin depositions. Because of the positive histology, no other immunohistochemical methods were used.

Postoperative echocardiography did not show any residual tricuspid regurgitation and improvement of the left ventricular systolic function with LVEF of $50 \%$ was observed. This probably followed the fast resorption of myxomatous emboli in the coronary circulation. The patient was discharged ten days after surgery, and a follow up in a cardiology outpatient clinic, was arranged. Echocardiographic screening of the first degree relatives has been organised.

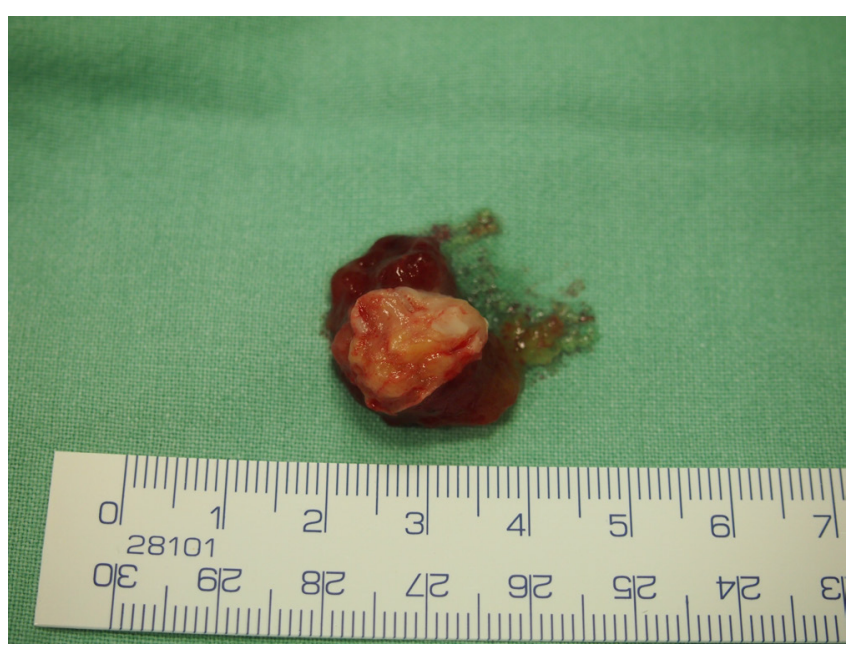

Fig. 6. Myxoma extirpated with adjacent atrial septum 


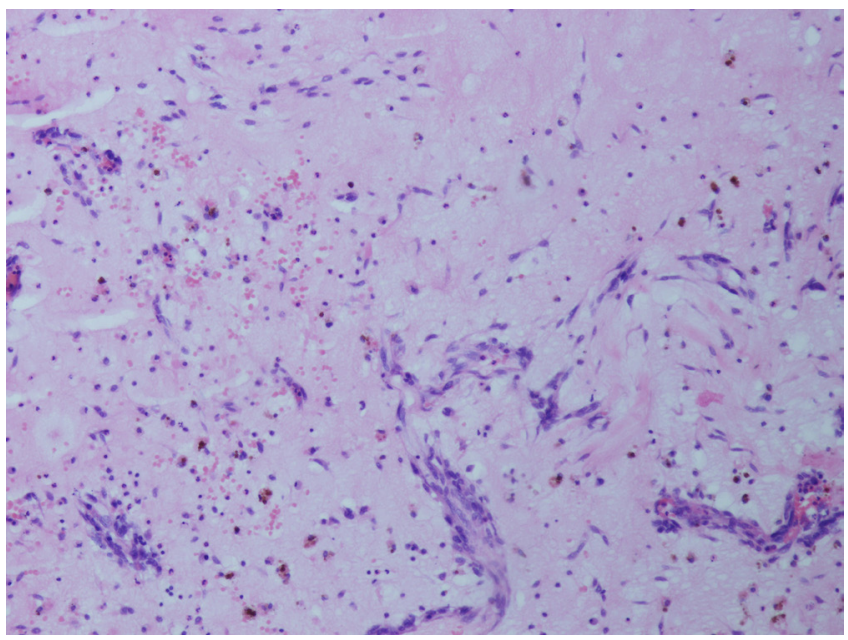

Fig. 7. Histopathological specimen showing myxoma tissue with reactive and regresive changes. H\&E 20x

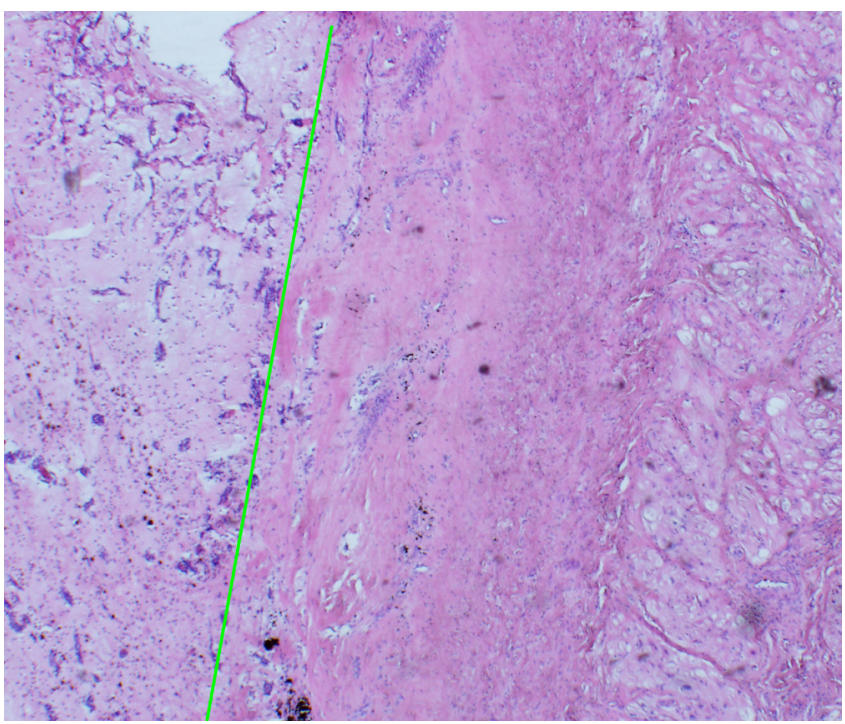

Fig 8. Histopathological specimen showing a border between myxoma tissue and myocardium. H\&E 100x

\section{DISCUSSION}

Heart tumours are rare with an estimated incidence of $0.002-0.02 \%$. They are benign in $75 \%$ of cases. Myxomas, first described by Virchow, are the most common benign heart tumours typically manifested in the $3^{\text {rd }}$ to $6^{\text {th }}$ decade of life, more often in women [5]. This type of tumour is considered to arise from multipotent mesenchymal cells which have persisted in the heart since the embryonal development period [6,7]. Cardiomyocyte progenitor cells and other poorly differentiated cells are another possible source. These cells are very often localised in the fossa ovalis area of the interatrial septum $[8,9]$. In $85 \%$ of cases, the tumour grows on the fibrovascular stalk attaching to the sub-endothelial base towards the left atrium. In $10 \%$ of patients, the myxoma grows towards the right atrium, and in the remaining $5 \%$ of patients, it is localised in the cavity of heart ventricles. Most commonly, myxomas are of a jelly consistency and 3-8 cm when diagnosed. Calcifications of left atrial myxomas are rarely reported, though they are described in more than fifty percent of right atrial myxomas [10]. Furthermore, focal haemorrhages are common. Approximately half of the myxomas have a smooth surface. The other half of myxomas have a fragile villous surface which has a greater potential to form embolisms. The embolic potential of myxoma is not proportional to its size but rather to it is related histological characteristics. Low fibrotic myxomas with a large myxoid intracellular mass, have a high embolic risk. On the other hand, highly fibrotic myxomas, more commonly found in elderly patients, have a lower rate of embolic complications [6].

In this reported case, the patient had a relatively small myxoma of little haemodynamic significance but with a high embolic potential because of its fragile villous surface. Histological examination showed a lot of myxoid intercellular matrix and regressive changes with haemorrhages and low grade of fibrillization.

In $4-10 \%$ of patients, myxomas are seen as a part of Carney complex [11]. Autosomal dominant inherited mutations in genes for PRKAR 1A and MYH9 synthesis are the probable cause. The other possible manifestations of the Carney complex are extracardiac myxomas and other tumours such as testicular tumours, schwannomas, pituitary adenomas and thyroid gland tumours as well as skin hyperpigmentation and endocrine disorders. Cardiac myxomas are often multiple and commonly atypically localised [12].

Regarding the risk of a familial transfer, routine echocardiographic screening for the first-degree relatives of patients with myxoma is recommended, particularly in young patients who have multiple or rightsided tumours.

Ninety percent of myxomas are visible on transthoracic echocardiography. An overall evaluation of the haemodynamic significance of myxoma especially interference with valvular apparatus is an important part of the examination.

Transesophageal echocardiography increases the sensitivity of myxoma detection up to $97 \%$ especially in cases of small myxomas, right atrial myxomas, or when a poor transthoracic window exists [13]. 
Other imaging modalities are indicated when the findings are unclear or atypical. Cardiac CT scan offers the advantage of a fast performance with an excellent three-dimensional resolution $(0.4-0.6 \mathrm{~mm})$. In a differential diagnosis, a CT scan compared to echocardiography is more reliable in excluding invasive growths of other malignant tumours into the cardiac or surrounding tissues $[14,15]$. A radiation burden is a disadvantage. A typical CT image of a myxoma is a spherical or ovoid heterogeneous tumour with a signal density lower than that of the myocardium. Less often, the signal density is the same but never higher than that of the myocardium [10]. Cardiovascular magnetic resonance (CMR) is another excellent diagnostic modality in the differential diagnostics of cardiac tumours [16]. It provides the advantages of best soft tissue resolution and absence of the radiation burden, but this is offset by the time required.

A myxoma is most often of a heterogeneous texture with lower signal intensity than blood in SSFP sequences on CMR imaging. Late gadolinium enhancement (LGE) is usually positive and often of a spotted character [17].

Clinical manifestation of a myxoma varies from being completely asymptomatic in $20 \%$ of cases, to a fully expressed triad of symptoms such as constitutional symptoms, signs of intracavitary heart obstruction and embolic complications which are reported in 30-45\% patients [4]. Fragments of a myxoma in the left heart and adhering blood clots can embolize anywhere in the systemic circulation and cause an infarction and eventually local haemorrhage. Half of myxoma embolisms from in the left heart are directed into cerebral arteries and are responsible for $0.5 \%$ of cerebrovascular accidents [18], and it is, therefore, necessary to exclude myxoma in cases of multiple cerebral artery emboli. Late development of vascular aneurysms at the site of adherent emboli is an associated unwanted risk of rupture and haemorrhage. Propagation of tumour cells in the vasa vasorum with destruction of the architecture of the arterial wall similarly to mycotic aneurysms is a possible explanation [19]. A direct tumour cell extension through the endothelium is another possible mechanism in the development of aneurysms.

Individual cases of embolisms into coronary arteries, retinal arteries, visceral arteries, aorta and peripheral arteries are also described in the literature.

Coronary artery embolisms are rare complications with an incidence of $0.06 \%$. The perpendicular direc- tion of the coronary arteries ostia from the aorta is a possible explanation for this $[20,21]$. Embolisms into the right coronary artery are more often described in the literature, in comparison with the left coronary artery. Patients typically present with STEMI rather than NSTEMI, but cases of sudden cardiac death have also been reported [22, 23].

In reality, the number of embolisms into coronary arteries may be higher because of the possibility of insufficient examination of all the patients with myocardial infarction or enduring sudden cardiac death. Embolism into an otherwise smooth coronary artery is a typical finding on coronary angiograms, though in about $25 \%$ of patients with proven embolic myocardial infarction, a standard angiogram is obtained and this may be explained by a fast resorption characteristic of the tumour in coronary arteries [24]. Pathological neovascularization of the myxoma on coronary angiogram is rarely observed [25].

The treatment of patients with an embolic acute myocardial infarction consists of the aspirations with catheters and balloon catheter dilatation if needed to achieve an opened artery. Thrombolytic therapy in patients with myxoma embolism is accompanied by a high risk of local bleeding [26-28].

Surgical extirpation is the only curative treatment possibility and is coupled with excellent results. Perioperative mortality is reported between $0-4 \%[29,30]$. A median sternotomy approach with extracorporeal circulation is common. Another possibility is a less invasive approach through a right mini-thoracotomy or partial hemi-sternotomy [31]. Complete extirpation of a myxoma with its fibrovascular stalk and the adjacent interatrial septum or the cardiac wall should be performed to prevent a local recurrence. The septal defect is then closed with a direct suture or with an autologous pericardium or synthetic patch. Surgical correction of other significant valvular pathology is then performed if necessary.

The long term prognosis following a successful surgery is excellent. Supraventricular tachyarrhythmias or a higher degree of atrioventricular block related to surgery can occur in some patients. A recurrence rate of $3 \%$ is reported, most often occurring in the first four years after the surgery $[2,10,30]$. Rare cases of extracardiac myxoma recurrences in the brain, lungs, kidneys, skeleton and skin, whose origins were probably from previous embolisms, have been reported [32, 33]. 


\section{CONCLUSION}

Embolism into the coronary artery from a left atrial myxoma is a rare cause of an acute myocardial infarction. Transesophageal echocardiography is indicated in all patients with an unclear source of coronary artery embolism. If a myxoma is found, early surgical resection is indicated. The surgery is of a curative nature with excellent long-time results. Appropriate dispensary care of patients after the surgery is necessary regarding the risk of possible myxoma recurrence.

\section{- CONFLICT OF INTEREST}

\section{None to declare.}

\section{- REFERENCES}

1. Obrenović-Kirćanski B. A 30-year-single-center experience in atrial myxomas: from presentation to treatment and prognosis. Thorac Cardiovasc Surg. 2013 Sep;61(6):530-6.

2. Amano J. Cardiac Myxoma: Its Origin and Tumor Characteristics. Ann Thorac Cardiovasc Surg. 2003(9):215-221.

3. Mendoza CE, Rosado MF, Bernal L: The role of interleukin- 6 in cases of cardiac myxoma. Clinical features, immunologic abnormalities, and a possible role in recurrence. Tex Heart Inst J. 2001;28:3.

4. Pinede $L$, Duhaut $P$, Loire R. Clinical presentation of left atrial cardiac myxoma. A series of 112 consecutive cases. Medicine (Baltimore). 2001;80(3):159-72.

5. Reynen K. Frequency of primary tumors of the heart. Am J Cardiol. 1996;77:107.

6. Burke AP, Tazelaar H, Gomez-Roman JJ, et al: Cardiac myxoma. In Travis WD, Brambilla E, Muller-Hermelink HK, Harris CC, editors. Pathology and Genetics of Tumours of the Lung, Pleura, Thymus and Heart. Lyon: IARC Press, 2004, pp. 260-3

7. Lie JT. The identity and histogenesis of cardiac myxomas: a controversy put to rest. Arch Pathol Lab Med. 1989;113:724-6.

8. Kodama H, Hirotani T, Suzuki $Y$, et al. Cardiomyogenic differentiation in cardiac myxoma expressing lineage-specific transcription factors. Am J Pathol. 2002;161:381-9.

9. Terracciano LM, Mhawech P, Suess K, et al. Calretinin as a marker for cardiac myxoma. Diagnostic and histogenetic considerations. Am J Clin Pathol. 2000;114:754-9.

10. Grebenc ML, Rosado-de-Christenson ML, Green $\mathrm{CE}$, et al. Cardiac myxoma: Imaging features in 83 patients. Radiographics. 2002;22:673.

11. Wilkes D, Charitakis K, Basson CT. Inherited disposition to cardiac myxoma development. Nat Rev Cancer. 2006;6:157.

12. Endo A, Ohtahara A, Kinugawa $T$, et al. Characteristics of cardiac myxoma with constitutional signs: a multicenter study in Japan. Clin Cardiol. 2002;25:367-70.

13. Meng Q, Lai H, Lima J, et al. Echocardiographic and pathologic characteristics of primary cardiac tumors: A study of 149 cases. Int J Cardiol. 2002;84:69.

14. Roberts WT, Bax JJ, Davies LC. Cardiac CT and CT coronary angiography: technology and application. Heart. 2008;94:78192.

15. van Beek EJ, Stolpen AH, Khanna G, et al. CT and MRI of pericardial and cardiac neoplastic disease. Cancer Imaging. 2007;7:19.

16. Pennell DJ, Sechtem UP, Higgins CB, et al. Clinical indications for cardiovascular magnetic resonance (CMR): Consensus Panel Report. Eur Heart J. 2004;25:1940-65.

17. Sparrow PJ, Kurian JB, Jones TR, Sivananthan MU. MR imaging of cardiac tumors. Radiographics. 2005;25:1255-76.

18. Knepper LE, Biller J, Adams HP Jr, Bruno A. Neurologic manifestations of atrial myxoma. A 12-year experience and review. Stroke. 1988;19:1435-144.

19. Herbs M. Cerebral Embolism from Left Atrial Myxoma Leading to Cerebral and Retinal Aneurysms: A Case Report. AJNR. 2005 26:666-669.

20. Lehrman KL, Prozan GB, Ullyot D. Atrial myxoma presenting as acute myocardial infarction. Am Heart J. 1985;110:1293-5.

21. Panos A, Kalangos A, Sztajzel J. Left atrial myxoma presenting with myocardial infarction. Case report and review of the literature. Int J Cardiol. 1997;62:73-5.

22. Kitchin PL, Benson P. Left Ventricular Myxoma Causing Sudden Death. The American Journal of Forensic Medicine and Pathology. 2015;36(2):58-60.

23. Braun S, Schrotter H, Reynen $\mathrm{K}$, et al. Myocardial infarction as complication of left atrial myxoma. Int J Cardiol. 2005;101:115121.

24. Chen RJ. Cardiac Myxoma With Unusual Obstructive and Embolic Presentation. Medicine (Baltimore). 2015; 94(38):e160.

25. Reynen K, Kockeritz U, Taha M, Strasser RH. Neovascularization in left atrial myxoma. Z Kardiol. 2004;93:69-71.

26. Kulkarni GB. Intravenous thrombolysis in patient with left atrial myxoma with acute ischemic stroke. Ann Indian Acad Neurol. 2014;17(4):455-58.

27. Sun MC. Intravenous Thrombolysis for Embolic Stroke due to Cardiac Myxoma. Case Rep Neurol. 2011; 3(1):21-26.

28. Abascal VM, Kasznica J, Aldea G, Davidoff R. Left atrial myxoma and acute myocardial infarction. A dangerous duo in the thrombolytic agent era. Chest. 1996;109:1106-8.

29. Ipek G, Erentug V, Bozbuga N, et al: Surgical management of cardiac myxoma. J Card Surg. 2005;20:300.

30. Samanidis G, Perreas K, Kalogris P, et al. Surgical treatment of primary intracardiac myxoma: 19 years of experience. Interact Cardiovasc Thorac Surg. 2011;13:597-600. 
Available online at: www.jccm.ro

31. Pineda AM., Santana O, Cortes-Bergoderi M, Lamelas J. Is a minimally invasive approach for resection of benign cardiac masses superior to standard full sternotomy?. Interact Cardiovasc Thorac Surg. 2013;16(6):875-79.

32. Aroca A, Mesa JM, Dominguez F, et al. Multiple recurrence of
The Journal of Critical Care Medicine 2017;3(3) • 117 a "sporadic" (non-familial) cardiac myxoma. Eur J Cardiothorac Surg. 1996;10:919-21.

33. Reber D, Birnbaum DE. Recurrent cardiac myxoma: why it occurs. A case report with literature review. J Cardiovasc Surg (Torino). 2001;42:345-8 